\title{
Symbol Timing Recovery for Generalized Minimum Shift Keying Modulations in Software Radio Receiver
}

\author{
Yik-Chung Wu and Tung-Sang Ng \\ Department of Electrical and Electronic Engineering \\ The University of Hong Kong \\ Pokfulam Road, Hong Kong \\ Tel.: ++ 852 + 28578406 Fax: ++ $852+25598738$
}

\begin{abstract}
A new symbol timing estimator for generalized MSK signals is proposed. It is based on the squaring algorithm and has a feedforward structure. The proposed timing estimator is fully-digital and is suitable for implementation in software radios. The performance in AWGN channel is compared with the Modified Cramer-Rao bound and that of the ML algorithm. The proposed timing estimator is found to have a performance close to that of the ML algorithm, but with a lower implementation complexity.
\end{abstract}

\section{INTRODUCTION}

Continuous phase modulation (CPM) [1] is a class of jointly power and bandwidth efficient digital modulation scheme. It has a constant envelope and therefore is very attractive to applications where nonlinear power amplifiers are employed to maximize power efficiency. However, the main drawback of CPM is that the optimum receiver for CPM, which is based on the Viterbi algorithm, requires high implementation complexity. Fortunately, there is a subset of CPM with binary data and modulation index equal to 0.5 , referred to as Generalized minimum shift keying (MSK) modulation [2][3], in which linear MSK-type receivers can be used with limited loss in performance compared to the Viterbi-algorithm based receivers.

Software radio has drawn much attention in recent years, as it is suitable for realizing multi-band multi-mode terminals. In software radio receiver, the digitalization point is shifted towards the antenna such that more and more functions are implemented in digital domain. Symbol timing recovery, which is a crucial part in demodulation, also has to be in all-digital form. Various all-digital timing recovery algorithms for generalized MSK signals have been reported in the literature [4]-[6]. In particular, the symbol timing recovery algorithm in [4] involves the transmission of a preamble, thereby lowering the transmission efficiency. The algorithm introduced in [5] is based on approximation of the Maximum Likelihood (ML) principle. Although it provides good performance, the implementation complexity is large. An ad hoc approach has been proposed in [6], but the performance is quite far away from the Modified Cramer-Rao bound.

In this paper, we propose a new all-digital symbol timing recovery algorithm for generalized MSK signals based on the

This work was supported by the Hong Kong Research Grants Council and by the University Research Committee of The University of Hong Kong, Hong Kong. squaring algorithm, which was originally designed for linear modulation [7]. The same algorithm has also been recently applied to GMSK signal by the authors [8]. The applicability of this algorithm to other members of generalized MSK modulations is addressed in this paper.

The paper is organized as follows. Section II gives an overview of the generalized MSK signal model and its linear approximation. Section III describes the proposed timing estimator. Simulation results are presented in section IV and conclusion is drawn in section $\mathrm{V}$.

\section{GENERALIZED MSK AND ITS LINEAR APPROXIMATION}

The complex envelope of the binary CPM is given by

$$
s(t)=\exp \left[j 2 \pi h \sum_{i=-\infty}^{\infty} a_{i} q(t-i T)\right] .
$$

In this expression, $a_{i}$ is the transmitted symbol, $h$ is the modulation index, $q(t)$ is given by $q(t)=\int_{-\infty}^{t} g(\tau) d \tau$ and $g(t)$ is a pulse over a finite interval $0 \leq t \leq L T$ satisfying $\int_{-\infty}^{\infty} g(\tau) d \tau=1 / 2$. Generalized MSK signal refers to the special case of $h=1 / 2$. With different $g(t)$, we have different modulations. Some of the popular modulations are raisedcosine (LRC), rectangular (LREC), Tamed Frequency Modulation (TFM) and Gaussian MSK (GMSK). Their corresponding pulses are given by

LRC: $\quad g(t)=\left\{\begin{array}{ll}\frac{1}{2 L T}\left[1-\cos \left(\frac{2 \pi t}{L T}\right)\right] & 0 \leq t \leq L T \\ 0 & \text { otherwise }\end{array}\right.$;

LREC: $\quad g(t)= \begin{cases}\frac{1}{2 L T} & 0 \leq t \leq L T \\ 0 & \text { otherwise }\end{cases}$

with MSK being the special case of $L=1$;

GMSK: $\quad g(t)=\frac{1}{2 T}\left[Q\left(2 \pi B \frac{t-T / 2}{\sqrt{\ln 2}}\right)-Q\left(2 \pi B \frac{t+T / 2}{\sqrt{\ln 2}}\right)\right]$

where $Q(t)=\int_{t}^{\infty}(2 \pi)^{-1 / 2} e^{-\tau^{2} / 2} d \tau$, and $B$ is the $3 \mathrm{~dB}$ bandwidth of the Gaussian filter; 
TFM: $\quad g(t)=\frac{1}{8}\left[g_{0}(t-T)+2 g_{0}(t)+g_{0}(t+T)\right]$

with $\quad g_{0}(t) \approx \sin \left(\frac{\pi t}{T}\right)\left[\frac{1}{\pi t}-\frac{2-\frac{2 \pi t}{T} \cot \left(\frac{\pi t}{T}\right)-\frac{\pi^{2} t^{2}}{T^{2}}}{\frac{24 \pi t^{3}}{T^{2}}}\right]$.

Laurent [9] has shown that any constant-amplitude binary phase modulation can be expressed as the sum of a finite number of time-limited amplitude modulated pulses. Specifically, (1) can be expressed as

$$
s(t)=\sum_{N=-\infty}^{\infty} \sum_{K=0}^{2^{L-1}-1} \exp \left[j \pi h A_{K, N}\right] C_{K}(t-N T)
$$

$$
\begin{array}{ll}
\text { where } & A_{K, N}=\sum_{n=-\infty}^{N} a_{n}-\sum_{i=1}^{L-1} a_{N-i} \alpha_{K, i}, \\
\text { and } & C_{K}(t)=S_{0}(t) \times \prod_{i=1}^{L-1} S_{i+L \cdot \alpha_{K, i}}(t) .
\end{array}
$$

In the above expression,

$$
\begin{gathered}
K=\sum_{i=1}^{L-1} 2^{i-1} \alpha_{K, i}, \\
S_{n}(t)=\frac{\sin [\Psi(t+n T)]}{\sin (h \pi)}, \\
\Psi(t)= \begin{cases}t \int_{-\infty}^{t} g(\tau) d \tau & t<L T \\
h \pi-\pi \int_{-\infty}^{t-L T} g(\tau) d \tau & t \geq L T .\end{cases}
\end{gathered}
$$

By plotting out all the component functions $C_{K}(t)$ for various generalized MSK modulations, it can be easily observed in every single case that the main pulse $C_{0}(t)$ conveys the most significant part of the energy of the signal. Table 1 shows the percentage of total energy that $C_{0}(t)$ contains for commonly used modulations. Therefore, it is reasonable to approximate the generalized MSK signal by the first component only. Assuming there is no data for $t<0$, we have

$$
\begin{aligned}
s(t) & \approx \sum_{N=0}^{\infty} \exp \left[j \pi h \sum_{n=0}^{N} a_{n}\right] C_{0}(t-N T) \\
& =\sum_{k=0}^{\infty} b_{2 k+1} C_{0}(t-2 k T-T)+j \sum_{k=0}^{\infty} b_{2 k} C_{0}(t-2 k T)
\end{aligned}
$$

where $b_{2 k}=b_{2 k-1} a_{2 k}, b_{2 k+1}=-b_{2 k} a_{2 k+1}$ and $b_{-1}=1$. Note that $C_{0}(t)$ is a pulse of length $(L+1) T$ and when $L=1$, the representation in (3) is exact as only $C_{0}(t)$ exists in this case.
Table 1.

The percentage of the total energy that the main pulse $C_{0}(t)$ contains

\begin{tabular}{|c|c|}
\hline Modulation pulse $g(t)$ & $\begin{array}{c}\text { Percentage of total energy that } C_{0}(t) \\
\text { contains }\end{array}$ \\
\hline 0.3GMSK & $99.6 \%$ \\
0.5GMSK & $99.96 \%$ \\
\hline 1REC (MSK) & $100 \%$ \\
2REC & $98.83 \%$ \\
3REC & $93.42 \%$ \\
\hline 1RC & $100 \%$ \\
2RC & $99.98 \%$ \\
\hline TFC & $99.43 \%$ \\
\hline
\end{tabular}

\section{THE PROPOSED TIMING ESTIMATOR}

We first consider a received linear modulation signal (PAM, QAM, PSK), with its complex envelope given by

$$
r(t)=\sum_{n=-\infty}^{\infty} a_{n} g_{T}(t-n T-\varepsilon T)+n(t),
$$

where $a_{n}$ is the $n^{\text {th }}$ data symbol, $g_{T}(t)$ is the transmission signal pulse, $T$ is the symbol duration, $n(t)$ is the white Gaussian noise with one-side power spectral density $N_{o}$ and $\varepsilon$ is an unknown delay but assumed slowly-varying. After the received signal passes through the RF front end, where out of band noise is rejected, $r(t)$ is sampled at a rate $1 / T_{s}=N / T$ and filtered by a digital receiving filter with impulse response $g_{R}(k T / N)$. The output of the digital receiving filter is given by

$$
\tilde{r}_{k}=\sum_{n=-\infty}^{\infty} a_{n} g(k T / N-n T-\varepsilon T)+\tilde{n}(k T / N),
$$

where $g(t)$ is the combined response of the symbol pulse and the receiving filter. $\tilde{n}(t)$ is the filtered noise.

Oerder and Meyr [7] proposed that the unknown timing delay can be estimated by computing the complex Fourier coefficient at the symbol rate for every segment of $L_{o} N$ samples ( $L_{o}$ symbols) of $\left|\tilde{r}_{k}\right|^{2}$. That is, the estimate is given by

$$
\hat{\varepsilon}_{m}=-\frac{1}{2 \pi} \times \arg \left(\sum_{k=m L_{o} N}^{(m+1) L_{o} N-1}\left|\tilde{r}_{k}\right|^{2} e^{-j 2 \pi k / N}\right) .
$$

In the frequency domain, the squaring operation is equivalent to self-convolution of the signal spectrum. The operation of self-convolution generates spectral lines at symbol rate $f= \pm l / T$, which contains the timing information. The accuracy of the estimate depends on the strength of the spectral lines generated at symbol rate, which in turn is determined by the degree of spectral overlap when the signal spectrums are $1 / T$ apart.

By exploiting the linear approximation in (3), a generalized MSK signal can be viewed as a combination of two orthogonal linear modulations each with symbol rate $1 / 2 T$ and staggered with a time $T$. Therefore, timing delays 
can be estimated separately in the inphase and quadrature channels by the squaring algorithm and the two estimates can be subsequently combined to give the timing delay estimate for the generalized MSK signal, $\hat{\varepsilon}$.

Denote the timing estimates of the inphase and quadrature branches in (3) by $\hat{\varepsilon}_{I}$ and $\hat{\varepsilon}_{Q}$ respectively. It can be shown [8] that, in the absence of estimation variance (in the case of high SNR or large $L_{o}$ ), $\left|\hat{\varepsilon}_{I}\right|+\left|\hat{\varepsilon}_{Q}\right|=0.5$. It implies that when the value of $\left|\hat{\varepsilon}_{I}\right|$ increases, $\left|\hat{\varepsilon}_{Q}\right|$ decreases, and vice versa. Based on this relationship, the proposed timing recovery method can be stated as:

$$
\hat{\varepsilon}=\left\{\begin{array}{lll}
2 \hat{\varepsilon}_{I} & \text { if } & \left|\hat{\varepsilon}_{I}\right| \leq 0.25 \\
2 \hat{\varepsilon}_{Q} & \text { if } & \left|\hat{\varepsilon}_{Q}\right|<0.25
\end{array}\right.
$$

There may be cases when both $\left|\hat{\varepsilon}_{I}\right|$ and $\left|\hat{\varepsilon}_{Q}\right|$ are very close to 0.25 , say $\left|\hat{\varepsilon}_{I}\right|=0.24$ and $\left|\hat{\varepsilon}_{Q}\right|=0.26$, and due to the variance of estimation, none of the case in (5) occurs. In this situation, either $\hat{\varepsilon}_{I}$ or $\hat{\varepsilon}_{Q}$ can be chosen to give the estimate of the generalized MSK signal as they both give a value close to 0.5 or -0.5 .

An obvious choice of the receiving filter for the proposed estimator is $H(f)=C_{0}(f)$, which matches to the pulse shape of the linear approximated generalized MSK signal. However, if $g(t)$ has a long pulse length $L$, the frequency spectrum of the main pulse $C_{0}(f)$ becomes narrow and therefore the degree of spectral overlap is reduced when signal spectrums are $1 / 2 T$ apart. As the strength of the spectral lines containing the timing information depends on the degree of spectral overlap, the strength of the spectral lines generated decreases and thus the estimator performance is degraded.

To counter this problem, an alternative receiving filter is proposed as $H(f)=R C_{4 T}(f) / C_{0}(f)$, where $R C_{4 T}(f)$ is the Fourier transform of a raised cosine pulse with $3 \mathrm{~dB}$ cutoff frequency $f_{c}=1 / 4 T$. By using this receiving filter, the signal spectrum that generates the spectral lines becomes $R C_{4 T}(f)$. There are two opposing effects when using this receiving filter. The first effect is that for long pulse length $L, R C_{4 T}(f)$ has stronger frequency components than $C_{0}(f)$ (as shown in Figure 1a for the case of 3REC), so that the degree of the spectral overlap is increased. On the other hand, as the maximum frequency span of $R C_{4 T}(f)$ is $\pm 0.5 / T$, this receiving filter zeros out all the frequency components of $C_{0}(f)$ for $|f|>0.5 / T$, which are originally used to obtain the timing information. Therefore, this receiving filter offers better performance than $H(f)=C_{0}(f)$ only for long-pulse-length cases. In these cases, the first effect is much stronger than the second one, resulting in an increase of spectral overlap. For the cases of short pulse length $L$, this receiving filter may not improve the performance and even cause degradation since the two effects may cancel out or even the second one becomes stronger than the first one (see Figure $1 \mathrm{~b}$ for the case of 1REC).

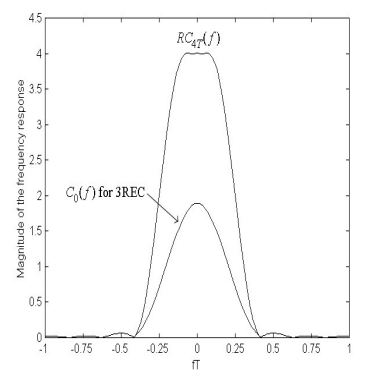

(a)

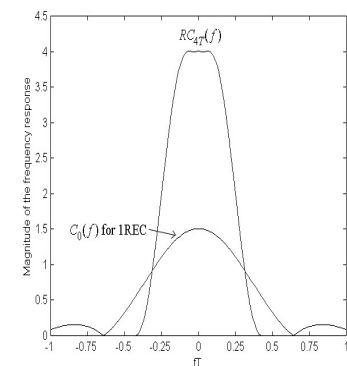

(b)
Figure 1. Magnitude of the frequency responses for (a) $C_{0}(f)$ of $3 R E C$ and $R C_{4 T}(f)$ (b) $C_{0}(f)$ of $1 \mathrm{REC}$ and $R C_{4 T}(f)$

\section{SIMULATIONS AND DISCUSSIONS}

The performances of the proposed algorithm for generalized MSK signals are assessed by simulation. Due to space limitation, only results of 1REC (MSK), 2RC and TFM are presented here. The variances of the estimates as a function of $E_{s} / N_{o}$ in AWGN channel is obtained by Monte Carlo simulation with each point averaged over $10^{4}$ estimates. In addition, the modified Cramer-Rao bound (MCRB) [10] and the performance of the estimator derived from the ML algorithm [5] are also shown for reference. In all simulations, the observation length $L_{o}$ is 64 and the oversampling factor $N$ is 4 .

Figure 2 shows the variance of the estimates for the case of 1REC. Let us first focus on the performance difference by using two different receiving filters. It is apparent that the proposed estimator using $C_{0}(f)$ as the receiving filter offers better performance. This is due to the fact that $C_{0}(f)$ contains significant frequency components for $|f|>0.5 / T$. Using $R C_{4 T}(f) / C_{0}(f)$ as the receiving filter zeros out most of the frequency components that originally yield the timing information in the squaring operation. Although $R C_{4 T}(f) / C_{0}(f)$ enhances some frequency components, the resultant performance is degraded.

Figure 3 depicts the performance of the proposed estimator for TFM. The results indicate that for TFM, using $R C_{4 T}(f) / C_{0}(f)$ as the receiving filter provides better performance. In contrast to the previous case, the frequency span of $C_{0}(f)$ for TFM is small due to its large value of $L$ ( $L=6$ in the simulation), $R C_{4 T}(f) / C_{0}(f)$ enhances the frequency components of $C_{0}(f)$ and thus give better performance.

Figure 4 illustrates the performance of the proposed estimator for $2 \mathrm{RC}$. In this case, the performances of the proposed estimator using two different receiving filters are similar. The two effects introduced by the receiving filter $R C_{4 T}(f) / C_{0}(f)$ almost cancel out and resultant degrees of spectral overlap using two different receiving filters are similar.

Now, let us compare the performance of the proposed estimator with the MCRB and that of the ML algorithm. From Figures 2-4, it is noted that, for the proposed timing estimator, there exists a receiving filter such that the performance is close to the MCRB. In comparison to the ML algorithm given in [5], two cases are considered separately. 
For pulse length $L \geq 2$ (Figure 3, 4), the proposed estimator exhibits only a limited degradation at low SNR, but the variance of estimates from the proposed estimator is smaller than that from [5] at high SNR. It is because in the derivation of the timing estimator based on the ML algorithm in [5], low SNR is assumed and performance in high SNR is sacrificed. For $L=1$ (Figure 2), the same observation can only be obtained for the receiving filter $C_{0}(f)$, as the receiving filter $R C_{4 T}(f) / C_{0}(f)$ degrades the performance of the proposed estimator in short-pulse-length cases, which has been explained earlier. However, what should be noted is that the performance curve of the proposed estimator with receiving filter $C_{0}(f)$ is parallel to the MCRB while that of the ML estimator exhibits an irreducible error floor at high SNR.

Despite a slight loss of performance at low SNR, the proposed algorithm has a lower implementation complexity, implying lower power consumption, which is an important advantage. Since it is known [11] that, the computation of complex Fourier coefficient at the symbol rate in (4) can be realized by an IIR filter with single coefficient of value -1 , the proposed algorithm requires only $2 L_{o} N$ real multiplications and $2 L_{o} N$ real additions per estimate. While the algorithm in [5] requires $3 L_{o} N+16$ real multiplications and $3 L_{o} N+12$ real additions per estimate, the saving in complexity of the proposed algorithm is about $33 \%$. In the calculation above, multiplication with -1 and 2 does not count.

\section{CONCLUSIONS}

An all-digital timing estimator for generalized MSK modulation based on squaring algorithm has been presented. The performance of the estimator in AWGN channel has been assessed by computer simulation. It has been shown that two receiving filters generate different performances in different modulations so that small variance of estimates can be guaranteed by using different receiving filters for different modulations. With lower implementation complexity, the performance of the proposed timing estimator is close to that of the ML estimator at low SNR and is better at high SNR.

\section{REFERENCES}

[1] C.-E. Sundberg, "Continuous phase modulation," IEEE Commun. Mag., pp. 25-38, Apr. 1986

[2] M. K. Simon, "A generalization of minimum shift keying (MSK)-type signaling based upon input data symbol pulse shaping," IEEE Trans. Commun., vol. 24, pp. 845-856, Aug. 1976.

[3] J. B. Anderson, T. Aulin, and C-E. Sundberg, "Digital Phase Modulation", New York: Plenum, 1986

[4] Y. L. Huang, K. D. Fan, C. C. Huang, "A fully digital noncoherent and coherent GMSK receiver architecture with joint symbol timing error and frequency offset estimation," IEEE Trans. Vehicular Tech., vol. 49, pp. 863-874, May 2000.

[5] M. Morelli and G. M. Vitetta, "Joint phase and timing synchronization algorithms for MSK-type signals," Proceeding of Mini-Conference of Communication Theory 1999, pp.146-150, Jun. 1999.

[6] M. Morelli and U. Mengali, "Joint frequency and timing recovery for MSK-type modulation," IEEE Trans. Commun., vol. 47, pp. 938-946, Jun. 1999.
[7] M. Oerder, H. Meyr, "Digital filter and square timing recovery," IEEE Trans. Commun., vol. 36, pp. 605-611, May 1988.

[8] Y. C. Wu and T. S. Ng, "Symbol timing recovery for GMSK modulation based on squaring algorithm," IEEE Commun. Letters, vol. 5, pp.221223, May 2001.

[9] P. A. Laurent, "Exact and approximate construction of digital phase modulations by superposition of amplitude modulated pulses (AMP)," IEEE Trans. Commun., vol. 34, pp. 150-160, Feb. 1986.

[10] U. Mengali and Aldo N. D'Andrea, "Synchronization techniques for digital receivers," New York : Plenum Press, c1997.

[11] M. Rahnema, "Symbol timing recovery and tracking method for burstmode digital communications," US Patent, Patent Number: 5870443.

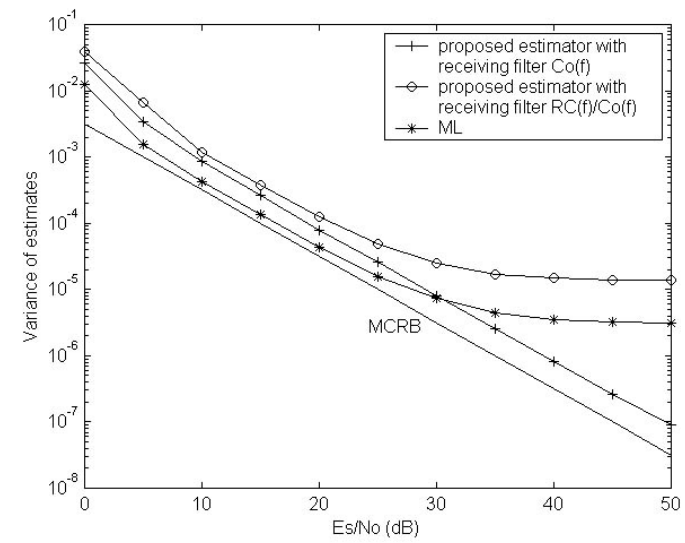

Figure 2. Estimation variance for 1REC (MSK)

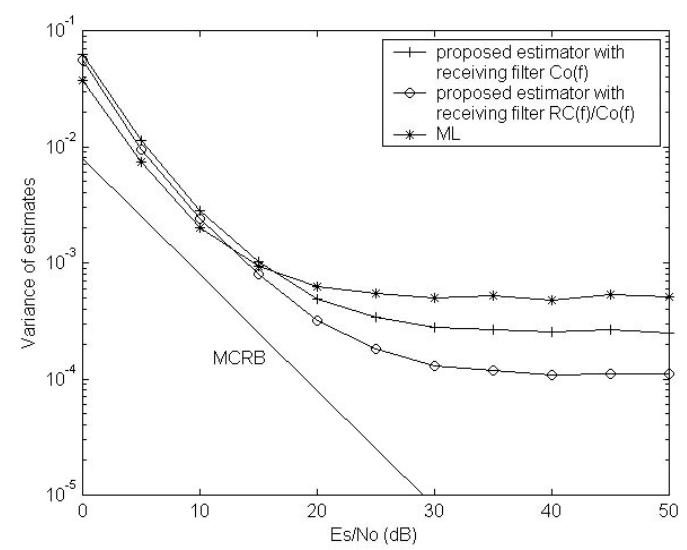

Figure 3. Estimation variance for TFM

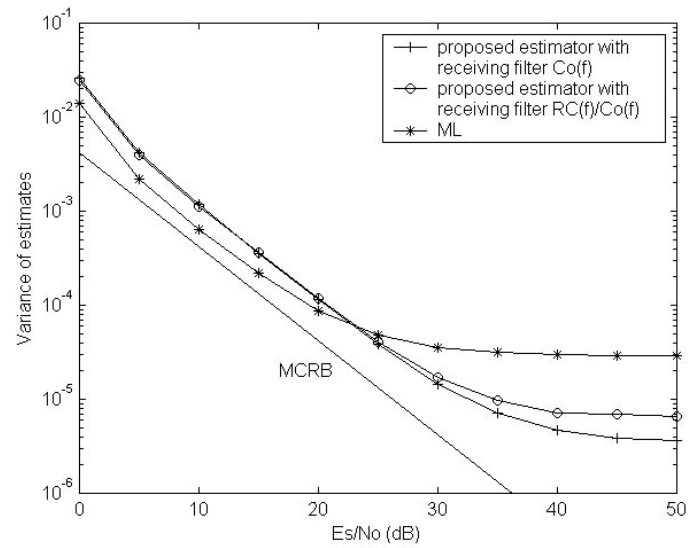

Figure 4. Estimation variance for $2 \mathrm{RC}$ 